\title{
ADPKD: Prototype of Cardiorenal Syndrome Type 4
}

\author{
Grazia Maria Virzì,, ${ }^{1}$ Valentina Corradi, ${ }^{1,2}$ Anthi Panagiotou, ${ }^{1,2}$ Fiorella Gastaldon, ${ }^{1}$ \\ Dinna N. Cruz, ${ }^{1,2}$ Massimo de Cal, ${ }^{1,2,3}$ Maurizio Clementi, ${ }^{4}$ and Claudio Ronco ${ }^{1,2}$ \\ ${ }^{1}$ Department of Nephrology, Dialysis and Transplantation, St. Bortolo Hospital, Via Rodolfi 37, 36100 Vicenza, Italy \\ ${ }^{2}$ IRRIV-International Renal Resarch Institute Vicenza, Vicenza, Italy \\ ${ }^{3}$ Division of Nephrology, Department of Medical and Surgical Sciences, University of Padova, 35128 Padova, Italy \\ ${ }^{4}$ Clinical Genetics unit, Department of Pediatrics, University of Padova, 35128 Padova, Italy \\ Correspondence should be addressed to Claudio Ronco, cronco@goldnet.it
}

Received 23 August 2010; Accepted 26 October 2010

Academic Editor: Mitchell H. Rosner

Copyright (๑) 2011 Grazia Maria Virzì et al. This is an open access article distributed under the Creative Commons Attribution License, which permits unrestricted use, distribution, and reproduction in any medium, provided the original work is properly cited.

\begin{abstract}
The cardiorenal syndrome type 4 (Chronic Renocardiac Syndrome) is characterized by a condition of primary chronic kidney disease (CKD) that leads to an impairment of the cardiac function, ventricular hypertrophy, diastolic dysfunction, and/or increased risk of adverse cardiovascular events. Clinically, it is very difficult to distinguish between CRS type 2 (Chronic Cardiorenal Syndrome) and CRS type 4 (Chronic Renocardiac Syndrome) because often it is not clear whether the primary cause of the syndrome depends on the heart or the kidney. Autosomal dominant polycystic kidney disease (ADPKD), a genetic disease that causes CKD, could be viewed as an ideal prototype of CRS type 4 because it is certain that the primary cause of cardiorenal syndrome is the kidney disease. In this paper, we will briefly review the epidemiology of ADPKD, conventional and novel biomarkers which may be useful in following the disease process, and prevention and treatment strategies.
\end{abstract}

\section{Introduction}

Heart performance and kidney function are closely interconnected, both in healthy and in disease conditions. It is also clear that there is a strong connection between renal and cardiovascular diseases. This bidirectional relationship between heart and kidney is physical, chemical, and biological. Primary disorders of one of these two organs often result in secondary dysfunction or injury to the other [1].

In this paper, we discuss about the ADPKD and its relation with cardiorenal syndrome. "Cardiorenal syndrome" (CRS) was defined as the pathophysiological disorder of the heart and kidney in which acute or chronic dysfunction in one organ may induce acute or chronic dysfunction in the other organ [2]. A large number of direct and indirect effects of each organ dysfunction can initiate and perpetuate the combined disorder of the two organs through a complex combination of neurohumoral feedback mechanisms [3]. For this reason, it was necessary to classify and divide the cardiorenal syndrome into different subtypes to provide a more concise and logically correct approach to this condition (see Table 1) [2].

Patients with CKD are at higher risk for cardiovascular events [4], and they have a 10- to 20-fold increased risk of cardiac death compared with age-gender-matched control subjects without CKD [5]. Part of this problem may be related to the fact that such individuals are also less likely to receive risk-modifying interventions compared to their nonCKD counterparts [6]. The association between reduced renal function and cardiovascular risk appears to consistently occur at estimated GFR levels below $60 \mathrm{ml} / \mathrm{min} / 1.73 \mathrm{~m}^{2}$ [7]. Clinically, it is very difficult to distinguish between CRS type 2 (Chronic Cardiorenal Syndrome) and CRS type 4 (Chronic Renocardiac Syndrome) because often it is not clear whether the primary cause of the syndrome depends on the heart or the kidney.

Autosomal dominant polycystic kidney disease, a genetic disease that causes CKD, could be viewed as an ideal prototype of CRS type 4 because it is certain that the kidney disease is the primary process. In this paper, we will briefly 
TABLE 1: Classification of cardiorenal syndorme (CRS).

\begin{tabular}{|c|c|c|}
\hline Acute cardiorenal syndrome & CRS type 1 & $\begin{array}{l}\text { Abrupt worsening of cardiac function leading to acute kidney injury } \\
\text { (AKI) }\end{array}$ \\
\hline Chronic cardiorenal syndrome & CRS type 2 & $\begin{array}{l}\text { Chronic abnormalities in cardiac function causing progressive } \\
\text { chronic kidney disease (CKD) }\end{array}$ \\
\hline Acute renocardiac syndrome & CRS type 3 & $\begin{array}{l}\text { Sudden worsening of renal function causing acute cardiac } \\
\text { dysfunction }\end{array}$ \\
\hline Chronic renocardiac syndrome & CRS type 4 & $\begin{array}{l}\text { Condition of primary CKD leading to an impairment of the cardiac } \\
\text { function (ventricular hypertrophy, diastolic dysfunction) and/or } \\
\text { increased risk of adverse cardiovascular events. }\end{array}$ \\
\hline Secondary cardiorenal syndrome & CRS type 5 & $\begin{array}{l}\text { Systemic disorders (e.g., sepsis) causing both cardiac and renal } \\
\text { dysfunction }\end{array}$ \\
\hline
\end{tabular}

review the epidemiology of $\mathrm{ADPKD}$, conventional and novel biomarkers which may be useful in following the disease process, and prevention and treatment strategies.

\section{Definition, Classification, and Epidemiology}

ADPKD occurs worldwide and in all races and ethnic groups [8]. It accounts for $\sim 10 \%$ of patients on renal replacement therapy representing an important cause of end-stage renal disease (ESRD) worldwide [9]. Prevalence of the disease is higher than that of Huntington disease, hemophilia, sickle cell disease, cystic fibrosis, myotonic dystrophy, and Down syndrome combined, and it occurs in approximately 1 of every 400 to 1000 live births [10]. Epidemiological data on the prevalence of ADPKD have been extensively reported, mainly in the United States and Europe. ADPKD is the fourth leading cause of CKD in the United States accounting for approximately $3 \%$ of cases [11]. In Europe, ADPKD as etiology of CKD Stage $\mathrm{V}$ has been reported as 7.8 and 6.0 per million for men and women, respectively [12]. With the advent of renal replacement therapy, cardiovascular complications have emerged as the major cause of death in ADPKD [13].

ADPKD is a genetically heterogeneous disease identified by two phenotypically similar forms associated with several mutations in two genes: the PKD1 gene located on chromosome $16(16 \mathrm{p} 13.3)$ and the PKD2 gene mapped to chromosome 4 (4q13-q23) [14]. A variety of genetic defects have been described in ADPKD patients, including deletions, frameshift, and missense mutations. Mutations of PKD1 gene, encoding the polycystin-1 protein, result in ADPKD type I (ADPKD1) which is responsible for approximately $85 \%$ of ADPKD cases. Gene PKD2 mutations, encoding the polycystin-2 protein, result in ADPKD type II (ADPKD2), corresponding to $15 \%$ of ADPKD cases [15].

Polycystin-1 is a large integral membrane protein with a domain architecture suggesting a function in cell-cell or cell-matrix interaction [16]. Polycystin-2 is a member of the calcium-permeable subfamily of transient receptor potential channels and forms a complex with polycystin-1 [17]. Polycystins are expressed in vascular smooth muscle and endothelia; it suggests a direct role of these proteins in the vascular manifestations of $\operatorname{ADPKD}[18,19]$. Both polycystin-1 and polycystin-2 are present in the primary cilium of tubular epithelial cells [20]. Mutations in these genes lead to abnormalities in cell proliferation, apoptosis, tubular basement membranes, and tubular fluid secretion, ultimately resulting in slowly expanding renal cysts [21].

The precise processes leading to cyst formation and loss of renal function remain incompletely understood. Several mechanisms contributing to the cyst formation have been identified, including a imbalance between epithelial cell proliferation and apoptosis, secretor defects, altered cellmatrix interactions, cell polarity, ciliary dysfunction, and altered intracellular signaling [22].

2.1. Clinical Presentation. Clinically, ADPKD is an adultonset disease characterized by progressive, bilateral renal cyst development and expansion of the kidneys [23]. Many patients with ADPKD are completely asymptomatic and often are diagnosed because of their positive family history or the development of hypertension (HP) [21]. Whether ADPKD2 patients are compared to ADPKD1, they seem to have a milder clinical presentation. Cysts and kidney failure occur at an earlier age in ADPKD1; the average age of CKD stage $\mathrm{V}$ is approximately 57 years in type I versus 69 years in type II [24]. ADPKD is a disease with a variable clinical course not only among families with different mutations, but within families with a defined mutation as well; it can be explained to the large extent by its genetic heterogeneity and modifier genes. Cysts may also develop in other organs. Liver cysts develop in more than $80 \%$ of patients, and the cysts are usually larger in women than in men [25]. Usually, cysts do not affect liver function. About $10 \%$ of patients have cysts in the pancreas, but these are functionally insignificant. Other locations of cysts include the spleen, arachnoid membranes, and seminal vesicles in men [26].

Hypertension is probably the most remediable and serious complication of ADPKD [10]. The new onset of $\mathrm{HP}$ in a patient at risk for polycystic kidney disease should prompt aggressive treatment and diagnostic studies. These patients during the course of ADPKD have early and more severe left ventricular hypertrophy (LVH). ADPKD may also occur with abdominal, back, and flank pain. Hematuria may be present secondary to cysts rupture. Urinary tract infections are also common.

A number of noncystic manifestations such as cardiovascular deficits, cardiac valve abnormalities, diverticular disease, and intracranial aneurysms are also associated with 
ADPKD; in fact, cardiovascular complications are the major cause of morbidity and mortality in patients with ADPKD [13].

2.2. Cardiovascular Complications. Mitral valve prolapsed (MVP) occurs in about $26 \%$ of affected adults compared with $2 \%$ of control subjects $[27,28]$. Aortic valve insufficiency can occur in association with the aortic root. Although these lesions may progress with time, they rarely need valve replacement. Screening echocardiography is not indicated unless a murmur is detected on examination [12]. Pericardial effusion may occur with an increased frequency in patients with ADPKD (35\% versus 9\% in a control group of patients with another chronic nephropathy), possibly as a result of increased compliance (or as a collagen protein dysfunction) of the parietal pericardium. Nevertheless, these effusions are generally well tolerated and clinically inconsequential. It is known that the kidney disease is strongly associated with a greater carotid Intima-Media Thickness (IMT). Subjects with ADPKD, even with preserved renal function, have a greater carotid IMT compared with healthy controls; carotid IMT is higher in hypertensive ADPKD patients [29].

It is challenging to clearly segregate those cardiovascular features due to the genetic disorder versus the secondary cardiovascular consequences of declining kidney function per se. However, recent improvement and expansion of genetically modified ADPKD animal models which mimic the human form are providing additional insights into the molecular mechanisms governing these disease processes as well as the development of cardiovascular complications. Several studies have provided convincing evidence that these vascular abnormalities are caused by alterations in the arterial wall linked to mutations in PKD1 or PKD2 [30].

Heterozygous mutant PKD1 or PKD2 mice appear normal but develop single cysts in the kidney or liver late in life and have a reduced overall lifespan [23, 31]. Homozygous null mutant mice are embryonically lethal and die in utero or perinatally because of systemic defects with massively enlarged cystic kidneys, pancreatic ductal cysts, and pulmonary hypoplasia and often exhibit edema, vascular leaks, and rupture of blood vessels. It suggests the role of polycystins for the structural integrity of blood vessels $[23,31,32]$.

In addition, most of the homozygous knockout embryos display multiple cardiac abnormalities including cardiac septation defects, double outlet right ventricle, and pericardial effusions [31, 33].

Moreover, Kurbegovic et al. engineered and described a Pkd1 transgenic mice (Pkd1(TAG) mice) that, in addition to the cystic phenotype, developed cardiac anomalies with severe left ventricular hypertrophy, marked aortic arch distention, and/or valvular stenosis and calcification [34].

Therefore, the cardiovascular complications seen in ADPKD patients begin to be recognized not only as a consequence of declining kidney function, but also as a defect due to the loss of polycystin-1 and/or polycystin-2 function in cardiovascular organs [31].
2.3. Hypertension and Left Ventricular Hypertrophy. As previously mentioned, hypertension and its consequent left ventricular hypertrophy are common in ADPKD patients, even in young adults, compared with unaffected controls [28]. Moreover, HP and LVH are associated with a faster progression to ESRD and an increased cardiovascular mortality $[35,36]$. It is well known that HP and LVH are associated with an accelerated rate of renal functional deterioration [37]. Both HP and LVH are important risk factors for cardiovascular death, the most frequent cause of mortality in ADPKD patients [13]; thus left ventricular hypertrophy may be considered a powerful indicator of mortality [38].

Hypertension in ADPKD occurs before the loss of kidney function in $60 \%$ of affected individuals and increases to almost $100 \%$ in patients with CKD stage IV-V $[39,40]$. The average age of onset of hypertension is 30-34 years [41], with men more commonly affected than women [42]. In added, its occurrence is earlier and more common in ADPKD1 than ADPKD2 patients [43]. The mechanisms leading to hypertension in ADPKD are not well understood.

However, it is now well known that increased activity of the intrarenal rather than the systemic renin-angiotensin system (RAS) is responsible for many forms of hypertension. Persistent elevation of intrarenal angiotensin II (ANG II) production with the inability to reduce ANG II in response to a high sodium intake will result in resetting the pressurenatriuresis relationship towards higher blood pressures leading to hypertension $[44,45]$.

Hypertension is associated with larger kidney size, reflecting a larger number of cysts and with the severity of kidney disease. Hypertensive ADPKD patients with normal kidney function show greater kidney volumes versus age-matched normotensive ADPKD men and women [41] increased proteinuria [46] and decreased renal blood flow [47]. Renal blood flow is reduced in hypertensive ADPKD patients versus matched essential hypertensive patients [47], and the renal resistive indices are also increased in hypertensive ADPKD subjects and are correlated with a loss of kidney function [43].

Renal structural changes play an important role in the pathogenesis of the HP, and renal arteriograms from end-stage ADPKD-nephrectomized specimens demonstrate marked attenuation of the vasculature due to the extrinsic compression by the presence of the cysts and their replacement by the latter [48]. Renal angiographic images of hypertensive ADPKD patients (from mild to advanced renal failure) show a large amount of a vascular renal substance peripheral to the outermost branches of the arterial tree [48].

Hypertensive ADPKD adults with normal kidney function show a greater frequency of LVH versus normotensive ADPKD men (50\% versus 30\%) and women (52\% versus $22 \%$ ) as well as with healthy controls [49]. They also show greater left ventricular mass index (LVMI) in comparison to matched essential hypertensive population $[43,50]$. The prevalence of $\mathrm{LVH}$ is increased even in the early stages of $\mathrm{CKD}$, and the frequency increases progressively as renal function decreases [51]. Several studies have shown increased LVMI, left ventricular diastolic dysfunction, endothelial dysfunction, and increased carotid IMT in young 
normotensive patients with ADPKD with well-preserved renal function. These findings suggest the cardiovascular involvement in the early stages during the course of ADPKD [26]. In experimental studies, hypertrophy was found not only in the left, but also in the right ventricle; these findings exclude that simple hemodynamic factors (increased preload and afterload) are the only explanation [51]. It is quite important to appreciate that hypertrophic remodeling comprises not only cardiomyocyte hypertrophy, but also interstitial fibrosis and microvessel disease [38].

2.4. Intracranial Aneurysms. A major complication of ADPKD includes the intracranial aneurysms (ICAs); the prevalence of ICA in patients with ADPKD ranges from 4 to $12 \%$, compared to a prevalence of $1 \%$ for the general population [52]. A familial clustering of ICA is found, with a 5 times greater chance of detecting an ICA in a subject with a relative with a ruptured ICA $[52,53]$; it suggests that genetic factors may be associated with the development of this complication [23]. This family clustering is in agreement with the finding that patients with mutations in the 5 ' region of PKD1 are more likely to have ICA than patients with $3^{\prime}$ mutations, especially in those with ICA rupture before 40 years old and in families with multiple cases of ICA or other vascular events [19].

Computed tomography, magnetic resonance angiography, and classical angiography are screening tests for detection of intracranial aneurysms in ADPKD subjects at high risk. The routine screening for asymptomatic ICA in all ADPKD individuals is not indicated, but in families with a proven case of ICA, a screening analysis is recommend in consultation with a neurosurgeon.

\section{Biomarkers}

In general, biomarkers can be divided into 3 subtypes based on the technical procedures used. Biomarkers measured by laboratory tests are defined as "laboratory or molecular biomarkers"; those related to signaling, imaging, and functional tests are defined as "functional biomarkers"; those related to genetic polymorphisms and other genomic tests are defined as "genetic biomarkers."

\subsection{Laboratory and Molecular Biomarkers}

3.1.1. Renal Biomarkers. In patients with ADPKD, a limited number of biomarkers have been investigated.

In a recent study, Meijer et al. investigated urinary biomarkers for different segments of the nephron in patients with ADPKD versus healthy controls. They choose urinary Immunoglobulin $\mathrm{G}$ ( IgG) as a marker of glomerular damage; urinary $\beta_{2}$-microglobulin (B2M), urinary kidney injury molecule 1 (KIM-1), N-acetyl- $\beta$-D-glucosammide (NAG), and neutrophil gelatinase-associated lipocalin (NGAL) as markers for damage of the proximal tubule; urinary hearttype fatty acid binding protein (HFABP) as a marker for damage of the distal tubule. Urinary macrophage migration inhibitory factor (MIF) and monocyte chemotatic protein 1 (MCP-1) were chosen as markers of inflammation.
The most important finding of this study is that excretion of all urinary biomarkers from all segments of the nephron was increased in patients with ADPKD compared with control subjects.

Furthermore, NGAL excretion was associated with renal blood flow and total renal volume independent of albuminuria. In addition, B2M and $\mathrm{H}$-FABP were associated inversely with measured GFR and effective renal blood flow independent of albuminuria; KIM-1, NGAL, and MCP-1 were associated positively with total renal volume independent of albuminuria [54].

In a recent paper, Bolignano et al. investigated and reported urinary and serum NGAL levels in a sample of 26 ADPKD patients. They found that urinary and serum NGAL levels were higher in ADPKD patients than in control subjects. They found a strong correlation with the glomerular filtration rate. In addiction, they divided patients into two groups according to the cystic development and kidney dimensions; subjects with higher cystic growth presented higher urinary and serum NGAL values with respect to others. They concluded that higher levels of NGAL are correlated to higher cystic growth and suggested that this protein could be also involved in the process of cystogenesis [55].

In 92 patients with ADPKD, Casal et al. reported a comparative study for three biomarker tests of early kidney damage such as urinary albumin and total $\beta-\mathrm{N}$ acetylhexosaminidase (Hex) and its isoenzymes (Hex A, Hex B), as well as serum glutathione peroxidase, which has been considered as a marker of renal proximal tubular function. They found a frequent elevation of the urinary Hex and an alteration of its isoenzymatic profile, with $31 \%$ of the normotensive ADPKD subjects with normoalbuminuria already presenting an increased proportion of Hex B isoenzyme. Furthermore, keeping age constant, they reported a partial significant correlation between the ultrasound score (kidney size and number of cysts) and the proportion of Hex $\mathrm{B}$, but not with albuminuria or cystatin $\mathrm{C}$. This confirms the hypothesis that tubular damage plays a role in the pathogenesis and progression of ADPDK [56].

Wong et al. valued measure GFR and serum cystatin C (Cys C) levels in 18 children with ADPKD versus 41 children with minor renal pathological states.

Serum creatinine levels did not differ between the ADPKD and control group, but GFR was significantly greater in the ADPKD group than in controls. Cys $C$ level for the ADPKD group was significantly lower than that of controls.

This study corroborates the increase of GFR in children and adolescents with ADPKD and the superior diagnostic performance of Cys $C$ [57]. In fact, in patients with CKD, Cys $\mathrm{C}$ was proposed to perform better as a marker of GFR than serum creatinine [58].

Apart from being a good marker for renal function, Cys $\mathrm{C}$ appears to be also a marker of cardiovascular risk in CRS types 2 and 4 and offers complementary prognostic information to other cardiac biomarkers like troponin $\mathrm{T}$ and NT-proBNP $[58,59]$. High concentrations of circulating Cys $\mathrm{C}$ have shown to be consistently and strongly associated with the cardiovascular outcomes [60]. 
Another index of ADPKD progression is microalbuminuria; in fact, there are numerous reports that have established that microalbuminuria is a frequent sign of kidney impairment in the disease, associated with a major cardiovascular risk. Microalbuminuria is present in patients with chronic heart failure (CHF) and progressive renal failure $[46,61]$. In ADPKD patients, microalbuminuria is associated with an increase in arterial pressure $[61,62]$ and progression to renal failure [62], as well as with a more severe cystic involvement [61]. Martinez-Vea et al. examined the prevalence of microalbuminuria in a normotensive ADPKD population. The study showed a high prevalence of microalbuminuria in this group and a tendency of these patients towards a greater systolic blood pressure, plasma renin activity, and left ventricular mass [61]. There are few information about renal alterations and vascular remodeling in ADPKD patients with normal or minimally increased levels of urine albumin excretion.

3.1.2. Cardiac Biomarkers. The natriuretic peptides (NPs) are a well-described family of hormones with a major role in sodium and body volume homeostasis [63]. BNP (brain natriuretic peptide), and NT-proBNP are correlated with the severity of heart failure (HF) and left ventricular (LV) function and are useful markers for diagnosis, management, and prognosis in patients with normal renal function. Recent studies indicated that both BNP and troponin $\mathrm{T}$ have a diagnostic power in patients with CKD to predict cardiovascular disease $[63,64]$. The NPs have shown prognostic utility in patients with various stages of renal insufficiency [65], demonstrating potential applications in CRS types 2 and 4. It is well established that patients with CKD have higher levels of both BNP and NT-proBNP than age- and gender-matched subjects without reduced renal function, even in the absence of clinical HF [66]. Thus, the relationship between BNP, renal function, and the severity of heart failure is less clear, and the association between NPs levels and renal function remains complex.

Cardiac troponin $\mathrm{T}$ (cTnT) is a specific marker for myocardial damage and a myocardial infarction. In hemodialysis patients, three large observational studies concordant with cTnT levels are associated strongly with the risk of incident for cardiovascular events [67-69]. Thus, increased $\mathrm{cTnT}$ levels represent a strong and independent predictor of global cardiovascular mortality in clinically stable hemodialysis patients. However, there are few studies describing the significance and the prognostic value of elevated serum cTnT levels in stable patients with moderate CKD [70].

At this time, the relationship between renal function and serum cTnT remains still unclear, and the significance of an increased cTnT concentration in patients with renal dysfunction remains controversial [71].

Unfortunately, there are currently no published data about specific cardiac biomarkers in ADPKD population.

3.2. Functional Biomarkers: Imaging Techniques (Table 2). Imaging techniques may enhance, extend, and refine our ability to diagnose and follow up cardiac and renal diseases.
3.2.1. Imaging Techniques for ADPKD Diagnosis. The main structural change seen for ADPKD is the formation of renal cysts; thus, it is evident that any enlargement of the cysts and the decrease in the volume of the renal parenchyma are the key factors in the progress of this disease [41]. Different imaging modalities such as Ultrasonography (US), Computed Tomography (CT), and Magnetic Resonance Imaging (MRI) have been used to quantify the size of the kidney in ADPKD. The consortium for radiologic imaging studies of polycystic kidney disease (CRISP) was created to develop innovative imaging techniques and analyses to follow disease progression or to evaluate treatments for ADPKD.

Ultrasonography was the earliest method used to measure kidney volume in vivo and has the advantage of being widely available and easily performed with modest cost in comparison with CT scan and MRI. Unfortunately, US cannot provide separate, reliable measurements of both the renal cyst volume and the renal parenchymal volume [72]. Individuals who are at risk for ADPKD are often screened by ultrasound using age-graded diagnostic criteria derived from individuals with mutations in PKD1 [73].

In families of unknown genotype, the presence of three or more (unilateral or bilateral) renal cysts is sufficient for establishing the diagnosis in individuals aged 15 to 39 years; two or more cysts in each kidney are sufficient for individuals aged 40 to 59 years, and four or more cysts in each kidney are required for older individuals aged 60 years. Conversely, less than two renal cysts in at-risk individuals aged $\geq 40$ years are sufficient to exclude the disease. US imaging does not provide a sufficiently certain diagnosis in at-risk individuals younger than 30 years of age; so the utility of this technique for disease exclusion is limited in younger subjects.

Families with mutations in PKD2 typically have less severe disease; in ADPKD2, the mild renal cystic involvement has an adverse impact on the sensitivity of US criteria that apply to diagnose the disease. As a result of reduced test sensitivity, the diagnostic criteria in use have a suboptimal performance for individuals with mutations in PKD2 [73].

For younger individuals in whom US might yield equivocal or indeterminate results, a negative CT scan or MRI may provide further assurance that they are unaffected. CT scan and MRI have greater sensitivity so that smaller cysts ( $\sim 2 \mathrm{~mm}$ compared with $\sim 10 \mathrm{~mm}$ for ultrasonography) can be detected [74]. Both CT and MRI avoid the potential pitfalls of US, that is, any operator-dependent techniques or the need for multiple image acquisitions of large kidneys. Contrast media-enhanced CT or electron beam CT techniques can provide accurate measurements not only of the total kidney volume, but also of the renal cyst volumes in ADPKD patients [75]. However, CT has two significant limitations: the radiation exposure and the requirement for administering intravenous contrast media. The contrast media may be associated with a small chance of serious allergic reactions and nephrotoxicity in patients with renal insufficiency.

MRI has increasingly been used because it provides highresolution 3D images with excellent tissue contrast without exposure to ionizing radiation or iodinated contrast medium 
TABLE 2: Imaging techniques.

Ultrasonography (US)

ADPKD diagnosis

Computed tomography (CT) scan

Magnetic resonance imaging (MRI)

Echocardiography

Diagnosis of cardiovascular complications

Transmitral pulsed doppler

Tissue doppler imaging (TDI)

Cardiovascular magnetic resonance imaging (cMRI)
Easy, available, and cheap method

Limited utility for disease exclusion in younger subjects and suboptimal performance for individuals with ADPKD type II

Detect small cysts $(\sim 2 \mathrm{~mm})$

Exposure to radiation and administration of intravenous contrast media

Provide high-resolution 3D images

No exposure to radiation, no administration of intravenous contrast media

Determine LV/RV size and function, LV wall motion abnormalities, valvular function and abnormalities, diastolic function, and presence or absence of pericardial abnormalities or intracardiac masses

Noninvasive method to evaluate the diastolic dysfunction

Influenced the loading condition of the left atria and heart rate.

Permit an assessment of myocardial motion, a sensitive index of ventricular relaxation

Noninvasive test

Gold standard for the assessment of ventricular dimensions.
[76]. While MRI is a reliable and precise method to measure renal volume, little information and data are available in the medical literature about the validity and accuracy of MRIbased kidney volume measurements in ADPKD patients $[41,76]$. Initial preliminary reports from the CRISP indicate that MRI is as least as accurate as CT scan for determining the rate of increase in kidney volume [41]. At the present time, MRI appears not appropriate for routine application. Some of the limitations of MRI include relatively long imageacquisition times and variability in the quality of images that can be produced from different MR scanners [76].

\subsubsection{Imaging Techniques for Diagnosis of Cardiovascular} Complications. Echocardiography determines left/right ventricular size and function, left ventricular wall motion abnormalities, valvular function and abnormalities, diastolic function, and presence or absence of pericardial abnormalities or intracardiac masses; it also evaluates intracardiac filling pressures.

Transmitral pulsed doppler is the classical noninvasive method of evaluation of diastolic dysfunction; it is influenced by a variety of factors such as the loading condition of the left atria and heart rate. Tissue doppler imaging (TDI) is a new technique that permits an assessment of myocardial motion, a sensitive index of ventricular relaxation, which is more independent of the hemodynamic condition and, therefore, a more reliable diastolic function index [77]. Unlike transmitral pulsed doppler, TDI directly measures the mechanical wall function by calculating the velocity of myocardial movement and has been shown to better monitoring diastolic function of the myocardium.
Starting as a research method little more than a decade ago, cardiovascular magnetic resonance imaging (cMRI) has rapidly evolved to become a powerful diagnostic tool used in routine clinical cardiology. CMRI provides a relatively novel method for accurate definition of cardiac dimensions and is accepted as the "gold standard" for the assessment of ventricular dimensions. Benefits of cMRI include the ability to obtain a great deal of information with one noninvasive test. CMRI is used for the assessment of regional and global ventricular function and to answer questions regarding anatomy. CMRI is able to assess ischemic versus nonischemic disease, infiltrative disease, valvular and congenital disorders, and hypertrophic disease, and determine viability. Unfortunately, there are currently no published data using cMRI in ADPKD patients.

3.3. Genetic Biomarkers. Genetic tests are the "gold standard" to screen individuals for ADPKD. Molecular genetic tests are helpful when imaging results are equivocal and/or when a definite diagnosis is required in a younger adult. There are 2 methods for ADPKD DNA testing: linkage analysis and direct mutation screening.

Presymptomatic testing is possible in larger families by linkage analysis using highly informative microsatellite markers flanking the PKD1 and PKD2 genes. A significant limit of linkage analysis is the need for a relatively large number of affected family members in order to establish the gene involved in the disease. In linkage analysis, the segregation of chromosomal markers is examined and compared within a family in whom the clinical status (affected or unaffected) of each individual is known. By 
examining several markers, a "haplotype" (a pattern of alleles on the same chromosome that are inherited together) that segregates with the disease can be determined [78]. There are several limitations to linkage testing; the most important is that no information can be obtained from testing the proband alone. Furthermore, linkage analysis cannot be used if a family is small, if family members refuse to participate, or if the proband is suspected to have a de novo mutation.

A direct mutation analysis is another method for molecular diagnosis in ADPKD. As ADPKD displays a high level of allelic heterogeneity, the complete screening of both genes is required. Most mutations are private (unique to a single family) and scattered throughout these genes with no clear "hot spots." Therefore, exon-by-exon screening of these genes is required to ensure a high sensitivity in detecting diseasecausing mutations, thus screening approaches are expensive. Screening of individuals with ADPKD detects mutations in up to $91 \%$ of cases. However, only approximately $65 \%$ of patients have definite mutations with approximately $26 \%$ having nondefinite changes that require further evaluation [74]. ADPKD database (Autosomal Dominant Polycystic Kidney Disease: Mutation Database) collects every known variants on $\mathrm{PKD} 1$ and $\mathrm{PKD} 2$ to improve the diagnostic value of molecular screening.

Furthermore, the recent availability of clinical molecular genetic testing means such testing may be applied to asymptomatic at-risk relatives of subjects with ADPKD. As with most renal diseases, early diagnosis with implementation of effective interventions has important implications and the best chance for preventing or slowing renal progression and cardiac complications in patients with the ADPKD [79].

For example, the early intervention of diagnosing and aggressively treating blood pressure in these patients, particularly blockade of the renin-angiotensin system, has the potential of preventing $\mathrm{LVH}$, cardiovascular complications, and mortality [79].

In that regard, Schrier et al. have reported that the age of ESRD in both men and women with ADPKD has increased in recent years and speculate that this effect has been associated with better blood pressure control and increased therapy with ACE inhibitors [80].

Other relevant indications for early identification of ADPKD include the provision of more detailed and specific information regarding prognosis and risk for complications, the ability to make more informed reproductive choices, motivation for enhanced compliance and medical followup, and the evaluation of living donors form affected families [81]. Screening is also important in clearing prospective living kidney donors from affected families [81]. Screening children under 18 years old is not strongly recommended because of the potential emotional and social impact of a positive diagnosis in these younger subjects. Before screening, counselling from experienced staff must be performed, in order to facilitate appropriate life-style decisions.

\section{Prevention, Management, and Trials}

The core of the prevention is that the reduction in the rate of progression of CKD may lead to a reduction of the incidence of chronic renocardiac syndrome. Many novel therapies have been evaluated in the cardiorenal syndrome setting, including agents that may block key local factors (e.g., adenosine $\mathrm{A}(\mathrm{I})$ receptor antagonists), improve diuresis, aquaresis, and natriuresis, and augment natural vasodilator mechanisms to improve renal perfusion [82].

There are no disease-specific therapies for any form of ADPKD, and no evidence-based guidelines on the management of ADPKD have been reported perhaps due to the very slow rate of disease progression. Interventions should be capable to slow down, stop, or reverse structural progression of the disease and should be able to prevent the decline of renal function improving clinical outcome. In ADPKD, only blood pressure control has been shown to have a favorable impact on disease progression and cardiovascular complication rate [83]. Rigorous control of blood pressure may prevent progression of renal disease and decreases the risk of cardiovascular morbidity that characterizes all patients with CKD. The KDOQI Clinical Practice guidelines on hypertension indicate that goal blood pressure should be less than 130/80 $\mathrm{mmHg}$ [84]. If there are no contraindications, an angiotensin-converting enzyme (ACE) inhibitor should be the initial antihypertensive agent. Increased reninangiotensin system activity and extracellular volume expansion play an important role in the pathogenesis of HP in patients with ADPKD, thus patients generally respond well to these agents [85]. ACE inhibitors or angiotensin receptor blockers (ARBs) may have renoprotective properties increasing renal blood flow which correlates with progression of ADPKD and contributes to cyst growth [12, 47, 86]. In a meta-analysis of 11 randomized controlled trials, Jafar et al. found that ACE inhibitors were more effective in lowering urine protein excretion in patients with ADPKD compared to regimes without ACE inhibitors, and it was more evident in patients with higher levels of proteinuria. However, the benefit of ACE inhibitors on ADPKD progression remains inconclusive [86].

Whether salt restriction and ACE inhibitors and ARBs therapy fail to lower blood pressure sufficiently, it may be necessary to add a diuretic (thiazides initially, with a switch to loop diuretics if thiazides are not effective) [25]. Additional agents may then be added to gain an appropriate blood pressure control and ameliorate other clinical advantages, such as angina using a $\beta$-blockers or calcium channel blockers. Addition of $\beta$-blockers to ACE inhibitor/ARB therapy is a very attractive choice, with documented cardiovascular protective characteristics. Several studies have shown better preservation of renal function or reduction in proteinuria and LVH with ACE inhibitors or ARBs compared to diuretics or calcium channel blockers $[87,88]$.

A controlled trial, the Halt Progression of Polycystic Kidney Disease study (NCT00283686), funded by the National Institutes of Health, is under way to determine whether treatment with ACE inhibitors and ARBs, administered singly or in combination, will reduce the rate of increase in kidney volume and slow the decline in GFR [25]. Significant advances in terms to understand the genetics of ADPKD and molecular mechanisms responsible for cyst initiation have revealed likely targets for therapeutic intervention. 
TABLE 3: Clinical trials for ADPKD.

\begin{tabular}{|c|c|c|c|c|}
\hline & $\begin{array}{l}\text { Start-finish } \\
\text { date }\end{array}$ & Intervention & Action & Design \\
\hline $\begin{array}{l}\text { HALT-ADPKD STUDY } \\
\text { (NCT00283686) }\end{array}$ & 2006-2013 & $\begin{array}{l}\text { ACE inhibitors and ARBs } \\
\text { (singly or in combination) }\end{array}$ & $\begin{array}{l}\text { Reduce the rate of } \\
\text { increase in kidney } \\
\text { volume and slow the } \\
\text { decline in GFR }\end{array}$ & $\begin{array}{l}\text { Multicenter, randomized, } \\
\text { placebo controlled }\end{array}$ \\
\hline NCT00428948 & 2007-2011 & $\begin{array}{l}\text { V2 receptor antagonist } \\
\text { (Tolvaptan) }\end{array}$ & $\begin{array}{l}\text { Reduce the } \\
\text { concentrations of cAMP } \\
\text { and slow the progression } \\
\text { of renal enlargement }\end{array}$ & $\begin{array}{l}\text { Multicenter, double blind, } \\
\text { placebo controlled }\end{array}$ \\
\hline NCT00309283 & 2006-2010 & $\begin{array}{l}\text { Long-acting somatostatin } \\
\text { (octreotide) }\end{array}$ & $\begin{array}{l}\text { Inhibit the growth of the } \\
\text { polycystic kidneys and } \\
\text { liver }\end{array}$ & $\begin{array}{l}\text { Randomized Single Center, } \\
\text { Single blind, Placebo } \\
\text { controlled }\end{array}$ \\
\hline NCT00426153 & $\begin{array}{l}2007-2010 \\
\text { (open-label } \\
\text { extention) }\end{array}$ & $\begin{array}{l}\text { Long-acting somatostatin } \\
\text { (octreotide) }\end{array}$ & $\begin{array}{l}\text { Inhibit the growth of the } \\
\text { polycystic kidneys and } \\
\text { liver }\end{array}$ & $\begin{array}{l}\text { Double blind, Randomized, } \\
\text { Placebo controlled, } \\
\text { Crossover }\end{array}$ \\
\hline NCT00565097 & $\begin{array}{l}\text { 2007-2009 } \\
\text { (open-label } \\
\text { extention) }\end{array}$ & $\begin{array}{l}\text { Long-acting somatostatin } \\
\text { (lanreotide) }\end{array}$ & $\begin{array}{l}\text { Inhibit the growth of the } \\
\text { polycystic kidneys and } \\
\text { liver }\end{array}$ & $\begin{array}{l}\text { Double blind, Randomized, } \\
\text { Placebo controlled }\end{array}$ \\
\hline $\begin{array}{l}\text { Trials with target } \\
\text { mTOR }\end{array}$ & - & $\begin{array}{l}\text { mTOR inhibitors } \\
\text { (sirolimus, everolimus) }\end{array}$ & $\begin{array}{l}\text { Modulate disease } \\
\text { progression and } \\
\text { development of renal } \\
\text { cysts }\end{array}$ & - \\
\hline
\end{tabular}

At the present time, there are no therapies proving a cyst progression delay and their complications, and there is no proven antihypertensive drug of choice neither in ADPKD patients nor in ADPKD patients on dialysis. An effective control of HP remains one of the few modifiable factors by medical intervention and may delay the development of LVH, which is strongly related with diastolic dysfunction [21]. A better understanding of the pathophysiology and the availability of animal models has enabled the development of preclinical trials and the identification of promising candidate drugs for clinical trials [12].

An hopeful therapeutic strategy, to inhibit cyst development in ADPKD, is modulating cyclic AMP (cAMP) levels. Arginine vasopressin (AVP) is a potent activator of renal adenyl cyclase [25]. The effect of AVP, via V2 Receptors, on cAMP levels in the collecting duct and distal nephron and the role of cAMP in cystogenesis provided the rationale for preclinical trials of vasopressin V2 receptor (VPV2R) antagonists [89]. In particular, one of these drugs, OPC31260 , reduces the concentrations of cAMP and inhibits cyst development in animal models of ADPKD [90]. An antagonist with high potency and selectivity for the human VPV2R (tolvaptan) has also been shown to be an effective treatment in PKD2 mouse model of autosomal dominant polycystic kidney disease [91]. The usefulness of AVP-V2 inhibitors in slowing the progression of renal enlargement and insufficiency in patients with ADPKD is currently evaluating in a placebo-controlled trial (NCT00428948) [25]. Small clinical trials (NCT00309283, NCT00426153, and NCT00565097) have shown that the administration of octreotide or lanreotide for a period of 6 to 12 months inhibits the growth of polycystic kidneys and livers [92-94].
Another promising therapeutic strategy might involve inhibitors of mTOR. The absence of polycystin permits excessive kinase activity in the mammalian which is the target of rapamycin (mTOR) pathway and the development of renal cysts [26]. The mTOR system can be blocked by rapamycin (sirolimus, everolimus), so it may be another possible strategy to modulate disease progression in ADPKD patients. Wahl et al. [95] found that inhibition of mTOR with rapamycin slows ADPKD progression and kidney enlargement in rats. In a prospective study in humans, rapamycin reduced polycystic liver volumes in ADPKD renal transplant recipients [96]. Larger studies of longer duration are needed to confirm the safety and to sustain efficacy of these novel treatments.

Experimental and clinical studies have suggested that statins may slow the progression of chronic kidney disease in general and ADPKD specifically [97]. Statins are widely used to lower cholesterol, and they have anti-inflammatory and antiproliferative qualities. However, there are some reported animal studies in Han:SPRD rats, an ADPKD model with many of the characteristics of the disease in humans, that demonstrate that statins reduce cyst formation and improve renal function [98].

Moreover, Namli et al. have shown in patients with ADPKD that statins have a beneficial effect in the reversal of endothelial dysfunction, an early manifestation of vascular injury. Six months of simvastatin therapy resulted in a significant improvement of endothelial dysfunction in patients with ADPKD. This finding may be in part related to the pleiotropic effects of simvastatin [99].

A clinical trail is currently in progress at the University of Colorado regarding ADPKD and pravastatin 
(NCT00456365) [100]. This study is designed to determine if the treatment with pravastatin can slow the progression of kidney and heart disease when initiated early in life in patients with ADPKD. This trial is expected to complete enrollment in 2011. The endpoints of interest in this threeyear study include total kidney volume and LVH index as measured MRI; urinary albumin excretion and endothelialdependent vasodilation as assessed by brachial ultrasound [100] (Table 3).

In conclusion, recent studies using different animal models of renal cystic diseases have suggested that various pharmacological interventions may modify disease progression. This clearly demonstrates that a better comprehension of the molecular and cellular defects underlying cystogenesis may lead to design novel therapeutic agents or a better use of existing ones. It is therefore likely that trials in human ADPKD will be carried out in the near future, especially as methods for assessing disease progression in the short term are now available $[41,101]$. Other important thing is that genetic counseling to discuss genetic risk, screening, and prenatal and predictive testing should be offered to all individuals with or at risk of inheriting ADPKD.

\section{Conflict of Interest}

No authors have reported a conflict of interest.

\section{References}

[1] L. W. Stevenson, A. Nohria, and L. Mielniczuk, "Torrent or torment from the tubules? Challenge of the cardiorenal connection," Journal of the American College of Cardiology, vol. 45, no. 12, pp. 2004-2007, 2005.

[2] C. Ronco, M. Haapio, A. A. House, N. Anavekar, and R. Bellomo, "Cardiorenal syndrome," Journal of the American College of Cardiology, vol. 52, no. 19, pp. 1527-1539, 2008.

[3] C. Ronco, C.-Y. Chionh, M. Haapio, N. S. Anavekar, A. House, and R. Bellomo, "The cardiorenal syndrome," Blood Purification, vol. 27, no. 1, pp. 114-126, 2009.

[4] G. M. Chertow, S.-L. T. Normand, L. R. Silva, and B. J. McNeil, "Survival after acute myocardial infarction in patients with end-stage renal disease: results from the cooperative cardiovascular project," American Journal of Kidney Diseases, vol. 35, no. 6, pp. 1044-1051, 2000.

[5] C. A. Herzog, "Dismal long-term survival of dialysis patients after acute myocardial infarction: can we alter the outcome?" Nephrology Dialysis Transplantation, vol. 17, no. 1, pp. 7-10, 2002.

[6] A. J. Collins, S. Li, D. T. Gilbertson, J. Liu, S.-C. Chen, and C. A. Herzog, "Chronic kidney disease and cardiovascular disease in the medicare population," Kidney International, vol. 64, no. 87, supplement, pp. S24-S31, 2003.

[7] C. Ronco, D. N. Cruza, and F. Ronco, "Cardiorenal syndromes," Current Opinion in Critical Care, vol. 15, no. 5, pp. 384-391, 2009.

[8] B. I. Freedman, J. M. Soucie, A. Chapman, J. Krisher, and W. M. McClellan, "Racial variation in autosomal dominant polycystic kidney disease," American Journal of Kidney Diseases, vol. 35, no. 1, pp. 35-39, 2000.

[9] S. Feng, G. M. Okenka, C.-X. Bai et al., "Identification and functional characterization of an $\mathrm{N}$-terminal oligomerization domain for polycystin-2," Journal of Biological Chemistry, vol. 283, no. 42, pp. 28471-28479, 2008

[10] T. Ecder and R. W. Schrier, "Cardiovascular abnormalities in autosomal-dominant polycystic kidney disease," Nature Reviews Nephrology, vol. 5, no. 4, pp. 221-228, 2009.

[11] USRDS 2008, August 2010, http://www.usrds.org/adr_2008 .htm.

[12] V. E. Torres, P. C. Harris, and Y. Pirson, "Autosomal dominant polycystic kidney disease," Lancet, vol. 369, no. 9569, pp. 1287-1301, 2007.

[13] G. M. Fick, A. M. Johnson, W. S. Hammond, and P. A. Gabow, "Causes of death in autosomal dominant polycystic kidney disease," Journal of the American Society of Nephrology, vol. 5, no. 12, pp. 2048-2056, 1995.

[14] S. Rossetti and P. C. Harris, "Genotype-phenotype correlations in autosomal dominant and autosomal recessive polycystic kidney disease," Journal of the American Society of Nephrology, vol. 18, no. 5, pp. 1374-1380, 2007.

[15] P. Igarashi and S. Somlo, "Genetics and pathogenesis of polycystic kidney disease," Journal of the American Society of Nephrology, vol. 13, no. 9, pp. 2384-2398, 2002.

[16] T. Mochizuki, G. Wu, T. Hayashi et al., "PKD2, a gene for polycystic kidney disease that encodes an integral membrane protein," Science, vol. 272, no. 5266, pp. 1339-1342, 1996.

[17] Y. Pei, A. D. Paterson, K. R. W. et al., "Bilineal disease and trans-heterozygotes in autosomal dominant polycystic kidney disease," American Journal of Human Genetics, vol. 68, no. 2, pp. 355-363, 2001.

[18] M. D. Griffin, V. E. Torres, J. P. Grande, and R. Kumar, "Vascular expression of polycystin," Journal of the American Society of Nephrology, vol. 8, no. 4, pp. 616-626, 1997.

[19] S. Rossetti, D. Chauveau, V. Kubly et al., "Association of mutation position in polycystic kidney disease 1 (PKD1) gene and development of a vascular phenotype," Lancet, vol. 361, no. 9376, pp. 2196-2201, 2003.

[20] F. Qian, A. Boletta, A. K. Bhunia et al., "Cleavage of polycystin-1 requires the receptor for egg jelly domain and is disrupted by human autosomal-dominant polycystic kidney disease 1-associated mutations," Proceedings of the National Academy of Sciences of the United States of America, vol. 99, no. 26, pp. 16981-16986, 2002.

[21] W. M. Bennett, "Autosomal dominant polycystic kidney disease: 2009 update for internists," Korean Journal of Internal Medicine, vol. 24, no. 3, pp. 165-168, 2009.

[22] P. D. Wilson, "Polycystic kidney disease," New England Journal of Medicine, vol. 350, no. 2, pp. 151-164, 2004.

[23] P. C. Harris and S. Rossetti, "Determinants of renal disease variability in ADPKD," Advances in Chronic Kidney Disease, vol. 17, no. 2, pp. 131-139, 2010.

[24] N. Hateboer, M. A.V. Dijk, N. Bogdanova et al., "Comparison of phonotypes of polycystic kidney disease types 1 and 2," Lancet, vol. 353, no. 9147, pp. 103-107, 1999.

[25] J. J. Grantham, "Clinical practice. Autosomal dominant polycystic kidney disease," New England Journal of Medicine, vol. 359, no. 14, pp. 1477-1485, 2008.

[26] W. E. Braun, "Autosomal dominant polycystic kidney disease: emerging concepts of pathogenesis and new treatments," Cleveland Clinic Journal of Medicine, vol. 76, no. 2, pp. 97104, 2009.

[27] M. Timio, C. Monarca, S. Pede, S. Gentili, C. Verdura, and S. Lolli, "The spectrum of cardiovascular abnormalities in autosomal dominant polycystic kidney disease: a 10-year follow-up in a five-generation kindred," Clinical Nephrology, vol. 37, no. 5, pp. 245-251, 1992. 
[28] D. D. Ivy, E. M. Shaffer, A. M. Johnson, W. J. Kimberling, A. Dobin, and P. A. Gabow, "Cardiovascular abnormalities in children with autosomal dominant polycystic kidney disease," Journal of the American Society of Nephrology, vol. 5, no. 12, pp. 2032-2036, 1995.

[29] O. Kocaman, H. Oflaz, E. Yekeler et al., "Endothelial dysfunction and increased carotid intima-media thickness in patients with autosomal dominant polycystic kidney disease," American Journal of Kidney Diseases, vol. 43, no. 5, pp. 854860, 2004.

[30] D. Bichet, D. Peters, A. J. Patel, P. Delmas, and E. Honoré, "Cardiovascular polycystins: insights from autosomal dominant polycystic kidney disease and transgenic animal models," Trends in Cardiovascular Medicine, vol. 16, no. 8, pp. 292-298, 2006.

[31] J. Stypmann, M. A. Engelen, S. Orwat et al., "Cardiovascular characterization of $\mathrm{Pkd} 2+/ \mathrm{LacZ}$ mice, an animal model for the autosomal dominant polycystic kidney disease type 2 (ADPKD2)," International Journal of Cardiology, vol. 120, no. 2, pp. 158-166, 2007.

[32] K. Kim, I. Drummond, O. Ibraghimov-Beskrovnaya, K. Klinger, and M. A. Arnaout, "Polycystin 1 is required for the structural integrity of blood vessels," Proceedings of the National Academy of Sciences of the United States of America, vol. 97, no. 4, pp. 1731-1736, 2000.

[33] C. Boulter, S. Mulroy, S. Webb, S. Fleming, K. Brindle, and R. Sandford, "Cardiovascular, skeletal, and renal defects in mice with a targeted disruption of the Pkdl gene," Proceedings of the National Academy of Sciences of the United States of America, vol. 98, no. 21, pp. 12174-12179, 2001.

[34] A. Kurbegovic, O. Côté, M. Couillard, C. J. Ward, P. C. Harris, and M. Trudel, "Pkd1 transgenic mice: adult model of polycystic kidney disease with extrarenal and renal phenotypes," Human Molecular Genetics, vol. 19, no. 7, pp. 1174-1189, 2010.

[35] T. Ecder and R. W. Schrier, "Hypertension in autosomaldominant polycystic kidney disease: early occurrence and unique aspects," Journal of the American Society of Nephrology, vol. 12, no. 1, pp. 194-200, 2001.

[36] A. B. Chapman, A. M. Johnson, S. Rainguet, K. Hossack, P. Gabow, and R. W. Schrier, "Left ventricular hypertrophy in autosomal dominant polycystic kidney disease," Journal of the American Society of Nephrology, vol. 8, no. 8, pp. 1292-1297, 1997.

[37] P. A. Gabow, A. M. Johnson, W. D. Kaehny et al., "Factors affecting the progression of renal disease in autosomaldominant polycystic kidney disease," Kidney International, vol. 41, no. 5, pp. 1311-1319, 1992.

[38] A. Remppis and E. Ritz, "Cardiac problems in the dialysis patient: beyond coronary disease," Seminars in Dialysis, vol. 21, no. 4, pp. 319-325, 2008.

[39] P. A. Gabow, D. W. Ikle, and J. H. Holmes, "Polycystic kidney disease: prospective analysis of nonazotemic patients and family members," Annals of Internal Medicine, vol. 101, no. 2, pp. 238-247, 1984.

[40] E. Valvo, L. Gammaro, N. Tessitore et al., "Hypertension of polycystic kidney disease: mechanisms and hemodynamic alterations," American Journal of Nephrology, vol. 5, no. 3, pp. 176-181, 1985.

[41] A. B. Chapman, L. M. Guay-Woodford, J. J. Grantham et al., "Renal structure in early autosomal-dominant polycystic kidney disease (ADPKD): The consortium for radiologic imaging studies of polycystic kidney disease (CRISP) cohort," Kidney International, vol. 64, no. 3, pp. 1035-1045, 2003.
[42] P. A. Gabow, "Medical progress: autosomal dominant polycystic kidney disease," New England Journal of Medicine, vol. 329, no. 5, pp. 332-342, 1993.

[43] A. B. Chapman, K. Stepniakowski, and F. Rahbari-Oskoui, "Hypertension in autosomal dominant polycystic kidney disease," Advances in Chronic Kidney Disease, vol. 17, no. 2, pp. 153-163, 2010.

[44] M. Loghman-Adham, C. E. Soto, T. Inagami, and L. Cassis, "The intrarenal renin-angiotensin system in autosomal dominant polycystic kidney disease," American Journal of Physiology, vol. 287, no. 4, pp. F775-F788, 2004.

[45] V. E. Torres, D. M. Wilson, J. C. Burnett Jr., C. M. Johnson, and K. P. Offord, "Effect of inhibition of converting enzyme on renal hemodynamics and sodium management in polycystic kidney disease," Mayo Clinic Proceedings, vol. 66, no. 10, pp. 1010-1017, 1991.

[46] A. B. Chapman, A. M. Johnson, P. A. Gabow, and R. W. Schrier, "Overt proteinuria and microalbuminuria in autosomal dominant polycystic kidney disease," Journal of the American Society of Nephrology, vol. 5, no. 6, pp. 1349-1354, 1994.

[47] A. B. Chapman, A. Johnson, P. A. Gabow, and R. W. Schrier, "The renin-angiotensin-aldosterone system and autosomal dominant polycystic kidney disease," New England Journal of Medicine, vol. 323, no. 16, pp. 1091-1096, 1990.

[48] I. H. H. T. Klein, G. Ligtenberg, P. L. Oey, H. A. Koomans, and P. J. Blankestijn, "Sympathetic activity is increased in polycystic kidney disease and is associated with hypertension," Journal of the American Society of Nephrology, vol. 12, no. 11, pp. 2427-2433, 2001.

[49] T. W. Doulton, A. K. Saggar-Malik, F. J. He et al., "The effect of sodium and angiotensin-converting enzyme inhibition on the classic circulating renin-angiotensin system in autosomal-dominant polycystic kidney disease patients," Journal of Hypertension, vol. 24, no. 5, pp. 939-945, 2006.

[50] A. Martinez-Vea, F. A. Valero, A. Bardaji et al., "Left ventricular hypertrophy in hypertensive patients with autosomal dominant polycystic kidney disease: Influence of blood pressure and humoral and neurohormonal factors," American Journal of Nephrology, vol. 20, no. 3, pp. 193-200, 2000.

[51] M.-L. Gross and E. Ritz, "Hypertrophy and fibrosis in the cardiomyopathy of uremia-beyond coronary heart disease," Seminars in Dialysis, vol. 21, no. 4, pp. 308-318, 2008.

[52] J. Huston III, V. E. Torres, P. P. Sulivan, K. P. Offord, and D. O. Wiebers, "Value of magnetic resonance angiography for the detection of intracranial aneurysms in autosomal dominant polycystic kidney disease," Journal of the American Society of Nephrology, vol. 3, no. 12, pp. 1871-1877, 1993.

[53] M. M. Belz, R. L. Hughes, W. D. Kaehny et al., "Familial clustering of ruptured intracranial aneurysms in autosomal dominant polycystic kidney disease," American Journal of Kidney Diseases, vol. 38, no. 4, pp. 770-776, 2001.

[54] E. Meijer, W. E. Boertien, F. L. Nauta et al., "Association of urinary biomarkers with disease severity in patients with autosomal dominant polycystic kidney disease: a crosssectional analysis," American Journal of Kidney Diseases, vol. 56, no. 5, pp. 883-895, 2010.

[55] D. Bolignano, G. Coppolino, S. Campo et al., "Neutrophil gelatinase-associated lipocalin in patients with autosomaldominant polycystic kidney disease," American Journal of Nephrology, vol. 27, no. 4, pp. 373-378, 2007. 
[56] J. A. Casal, J. Hermida, X. M. Lens, and J. C. Tutor, "A comparative study of three kidney biomarker tests in autosomaldominant polycystic kidney disease," Kidney International, vol. 68, no. 3, pp. 948-954, 2005.

[57] H. Wong, L. Vivian, G. Weiler, and G. Filler, "Patients with autosomal dominant polycystic kidney disease hyperfiltrate early in their disease," American Journal of Kidney Diseases, vol. 43, no. 4, pp. 624-628, 2004.

[58] D. N. Cruz, S. Soni, L. Slavin, C. Ronco, and A. Maisel, "Biomarkers of cardiac and kidney dysfunction in cardiorenal syndromes," Contributions to Nephrology, vol. 165, pp. 83-92, 2010.

[59] S. Manzano-Fernández, M. Boronat-Garcia, M. D. Albaladejo-Otón et al., "Complementary prognostic value of cystatin C, N-terminal Pro-B-type natriuretic peptide and cardiac troponin $\mathrm{T}$ in patients with acute heart failure," American Journal of Cardiology, vol. 103, no. 12, pp. 1753-1759, 2009.

[60] Y. Iwanaga and S. Miyazaki, "Heart failure, chronic kidney disease, and biomarkers - an integrated viewpoint," Circulation Journal, vol. 74, no. 7, pp. 1275-1282, 2010.

[61] A. Martinez-Vea, C. Gutierrez, A. Bardají et al., "Microalbuminuria in normotensive patients with autosomal-dominant polycystic kidney disease," Scandinavian Journal of Urology and Nephrology, vol. 32, no. 5, pp. 356-359, 1998.

[62] A. Martinez-Vea, A. Bardají, C. Gutierrez et al., "Exercise blood pressure, cardiac structure, and diastolic function in young normotensive patients with polycystic kidney disease: a prehypertensive state," American Journal of Kidney Diseases, vol. 44, no. 2, pp. 216-223, 2004.

[63] L. B. Daniels and A. S. Maisel, "Natriuretic peptides," Journal of the American College of Cardiology, vol. 50, no. 25, pp. 2357-2368, 2007.

[64] C. R. DeFilippi, J. C. Fink, C. M. Nass, H. Chen, and R. Christenson, "N-Terminal pro-B-type natriuretic peptide for predicting coronary disease and left ventricular hypertrophy in asymptomatic CKD not requiring dialysis," American Journal of Kidney Diseases, vol. 46, no. 1, pp. 35-44, 2005.

[65] S. J. Carr, S. Bavanandan, B. Fentum, and L. Ng, "Prognostic potential of brain natriuretic peptide (BNP) in predialysis chronic kidney disease patients," Clinical Science, vol. 109, no. 1, pp. 75-82, 2005.

[66] P. A. McCullough and K. R. Sandberg, "Sorting out the evidence on natriuretic peptides," Reviews in Cardiovascular Medicine, vol. 4, supplement 4, pp. S13-S19, 2003.

[67] D. S. Ooi, D. Zimmerman, J. Graham, and G. A. Wells, "Cardiac troponin T predicts long-term outcomes in hemodialysis patients," Clinical Chemistry, vol. 47, no. 3, pp. 412-417, 2001.

[68] J. Dierkes, U. Domröse, S. Westphal et al., "Cardiac troponin $\mathrm{T}$ predicts mortality in patients end-stage renal disease," Circulation, vol. 102, no. 16, pp. 1964-1969, 2000.

[69] F. Mallamaci, C. Zoccali, S. Parlongo et al., "Troponin is related to left ventricular mass and predicts all-cause and cardiovascular mortality in hemodialysis patients," American Journal of Kidney Diseases, vol. 40, no. 1, pp. 68-75, 2002.

[70] G. N. I. Wood, B. Keevil, J. Gupta et al., "Serum troponin $\mathrm{T}$ measurement in patients with chronic renal impairment predicts survival and vascular disease: a 2 year prospective study," Nephrology Dialysis Transplantation, vol. 18, no. 8, pp. 1610-1615, 2003.
[71] T. Tsutamoto, C. Kawahara, M. Yamaji et al., "Relationship between renal function and serum cardiac troponin $\mathrm{T}$ in patients with chronic heart failure," European Journal of Heart Failure, vol. 11, no. 7, pp. 653-658, 2009.

[72] R. L. Young and K.-B. Lee, "Reliability of magnetic resonance imaging for measuring the volumetric indices in autosomaldominant polycystic kidney disease: correlation with hypertension and renal function," Nephron Clinical Practice, vol. 103, no. 4, pp. c173-c180, 2006.

[73] Y. Pei, J. Obaji, A. Dupuis et al., "Unified criteria for ultrasonographic diagnosis of ADPKD," Journal of the American Society of Nephrology, vol. 20, no. 1, pp. 205-212, 2009.

[74] P. C. Harris and S. Rossetti, "Molecular diagnostics for autosomal dominant polycystic kidney disease," Nature Reviews Nephrology, vol. 6, no. 4, pp. 197-206, 2010.

[75] B. F. King, J. E. Reed, E. J. Bergstralh, P. F. Sheedy II, and V. E. Torres, "Quantification and longitudinal trends of kidney, renal cyst, and renal parenchyma volumes in autosomal dominant polycystic kidney disease," Journal of the American Society of Nephrology, vol. 11, no. 8, pp. 1505-1511, 2000.

[76] K. T. Bae, C. Tao, F. Zhu et al., "MRI-based kidney volume measurements in ADPKD: reliability and effect of gadolinium enhancement," Clinical Journal of the American Society of Nephrology, vol. 4, no. 4, pp. 719-725, 2009.

[77] A. D. Waggoner and S. M. Bierig, "Tissue Doppler imaging: a useful echocardiographic method for the cardiac sonographer to assess systolic and diastolic ventricular function," Journal of the American Society of Echocardiography, vol. 14, no. 12, pp. 1143-1152, 2001.

[78] W. Burke, "Genetic testing," The New England Journal of Medicine, vol. 347, no. 23, pp. 1867-1875, 2002.

[79] M. Taylor, A. M. Johnson, M. Tison, P. Fain, and R. W. Schrier, "Earlier diagnosis of autosomal dominant polycystic kidney disease: importance of family history and implications for cardiovascular and renal complications," American Journal of Kidney Diseases, vol. 46, no. 3, pp. 415-423, 2005.

[80] R. W. Schrier, K. K. McFann, and A. M. Johnson, "Epidemiological study of kidney survival in autosomal dominant polycystic kidney disease," Kidney International, vol. 63, no. 2, pp. 678-685, 2003.

[81] R. D. Perrone and D. C. Miskulin, "Hypertension in individuals at risk for autosomal dominant polycystic kidney disease: to screen or not to screen?" American Journal of Kidney Diseases, vol. 46, no. 3, pp. 557-559, 2005.

[82] H. Krum, P. Iyngkaran, and S. Lekawanvijit, "Pharmacologic management of the cardiorenal syndrome in heart failure," Current Heart Failure Reports, vol. 6, no. 2, pp. 105-111, 2009.

[83] R. Schrier, K. McFann, A. Johnson et al., "Cardiac and renal effects of standard versus rigorous blood pressure control in autosomal-dominant polycystic kidney disease: results of a seven-year prospective randomized study," Journal of the American Society of Nephrology, vol. 13, no. 7, pp. 1733-1739, 2002.

[84] A. S. Levey, M. V. Rocco, S. Anderson et al., "K/DOQI clinical practice guidelines on hypertension and antihypertensive agents in chronic kidney disease," American Journal of Kidney Diseases, vol. 43, no. 5, supplement 1, pp. S1-S290, 2004.

[85] R. W. Schrier, "Renal volume, renin-angiotensin-aldosterone system, hypertension, and left ventricular hypertrophy in patients with autosomal dominant polycystic kidney disease," Journal of the American Society of Nephrology, vol. 20, no. 9, pp. 1888-1893, 2009. 
[86] T. H. Jafar, P. C. Stark, C. H. Schmid et al., "The effect of angiotensin-converting-enzyme inhibitors on progression of advanced polycystic kidney disease," Kidney International, vol. 67, no. 1, pp. 265-271, 2005.

[87] K. Nutahara, E. Higashihara, S. Horie et al., "Calcium channel blocker versus angiotensin II receptor blocker in autosomal dominant polycystic kidney disease," Nephron Clinical Practice, vol. 99, no. 1, pp. c18-c23, 2005.

[88] T. Ecder, C. L. Edelstein, G. M. Fick-Brosnahan et al., "Diuretics versus angiotensin-converting enzyme inhibitors in autosomal dominant polycystic kidney disease," American Journal of Nephrology, vol. 21, no. 2, pp. 98-103, 2001.

[89] V. E. Torres, "Treatment strategies and clinical trial design in ADPKD," Advances in Chronic Kidney Disease, vol. 17, no. 2, pp. 190-204, 2010.

[90] V. E. Torres, X. Wang, Q. Qian, S. Somlo, P. C. Harris, and V. H. Gattone II, "Effective treatment of an orthologous model of autosomal dominant polycystic kidney disease," Nature Medicine, vol. 10, no. 4, pp. 363-364, 2004.

[91] V. E. Torres, "Role of vasopressin antagonists," Clinical Journal of the American Society of Nephrology, vol. 3, no. 4, pp. 1212-1218, 2008.

[92] A. Caroli, L. Antiga, M. Cafaro et al., "Reducing polycystic liver volume in ADPKD: effects of somatostatin analogue octreotide," Clinical Journal of the American Society of Nephrology, vol. 5, no. 5, pp. 783-789, 2010.

[93] M. C. Hogan, T. V. Masyuk, L. J. Page et al., "Randomized clinical trial of long-acting somatostatin for autosomal dominant polycystic kidney and liver disease," Journal of the American Society of Nephrology, vol. 21, no. 6, pp. 1052-1061, 2010.

[94] L. van Keimpema, F. Nevens, R. Vanslembrouck et al., "Lanreotide reduces the volume of polycystic liver: a randomized, double-blind, placebo-controlled trial," Gastroenterology, vol. 137, no. 5, article e1-2, pp. 1661-1668, 2009.

[95] P. R. Wahl, A. L. Serra, M. Le Hir, K. D. Molle, M. N. Hall, and R. P. Wüthrich, "Inhibition of mTOR with sirolimus slows disease progression in Han:SPRD rats with autosomal dominant polycystic kidney disease (ADPKD)," Nephrology Dialysis Transplantation, vol. 21, no. 3, pp. 598-604, 2006.

[96] Q. Qian, H. Du, B. F. King et al., "Sirolimus reduces polycystic liver volume in ADPKD patients," Journal of the American Society of Nephrology, vol. 19, no. 3, pp. 631-638, 2008.

[97] R. G. Fassett, J. S. Coombes, D. Packham, K. F. Fairley, and P. Kincaid-Smith, "Effect of pravastatin on kidney function and urinary protein excretion in autosomal dominant polycystic kidney disease," Scandinavian Journal of Urology and Nephrology, vol. 44, no. 1, pp. 56-61, 2010.

[98] F. A. Belibi and C. L. Edelstein, "Novel targets for the treatment of autosomal dominant polycystic kidney disease," Expert Opinion on Investigational Drugs, vol. 19, no. 3, pp. 315-328, 2010.

[99] S. Namli, H. Oflaz, F. Turgut et al., "Improvement of endothelial dysfunction with simvastatin in patients with autosomal dominant polycystic kidney disease," Renal Failure, vol. 29, no. 1, pp. 55-59, 2007.

[100] Clinical Trials., October 2010, http://www.clinicaltrials.gov/.

[101] B. F. King, V. E. Torres, M. E. Brummer et al., "Magnetic resonance measurements of renal blood flow as a marker of disease severity in autosomal-dominant polycystic kidney disease," Kidney International, vol. 64, no. 6, pp. 2214-2221, 2003. 


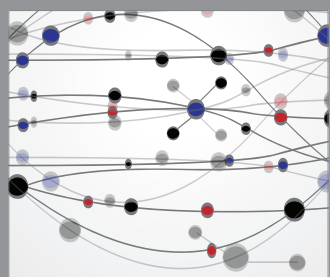

The Scientific World Journal
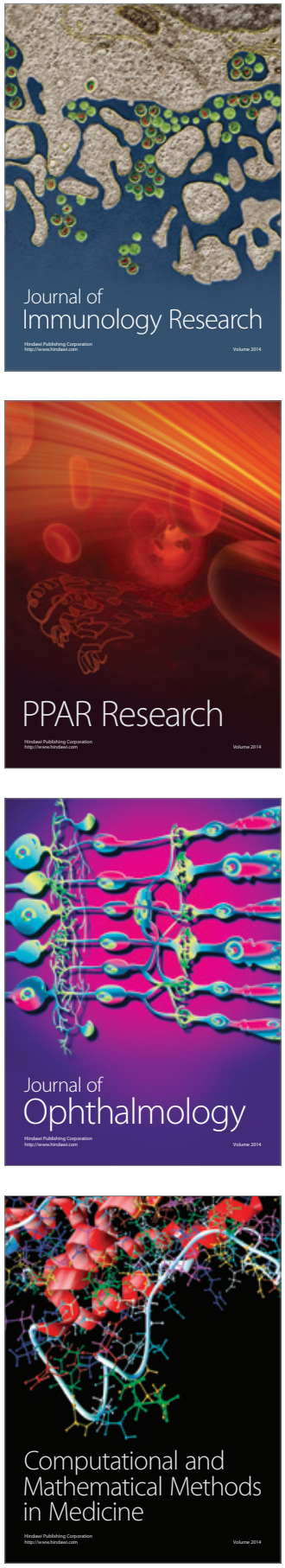

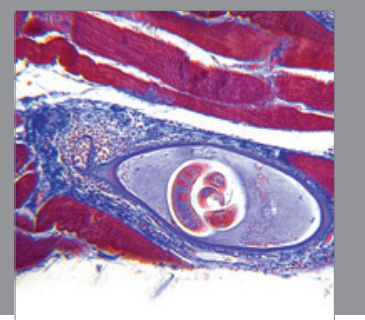

Gastroenterology

Research and Practice
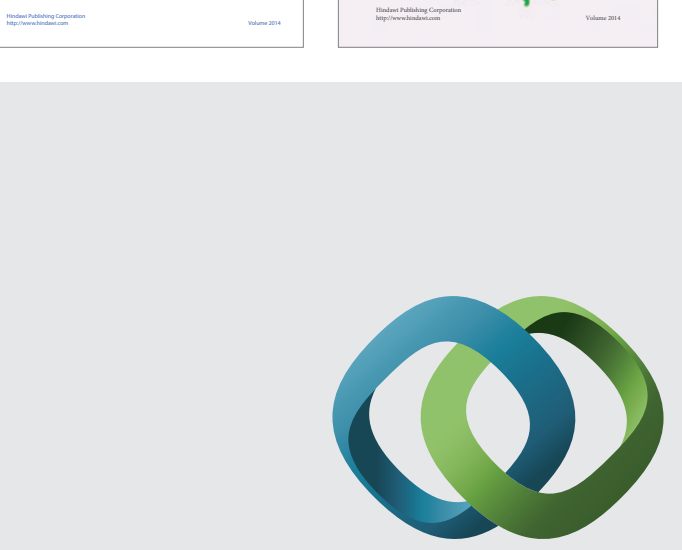

\section{Hindawi}

Submit your manuscripts at

http://www.hindawi.com
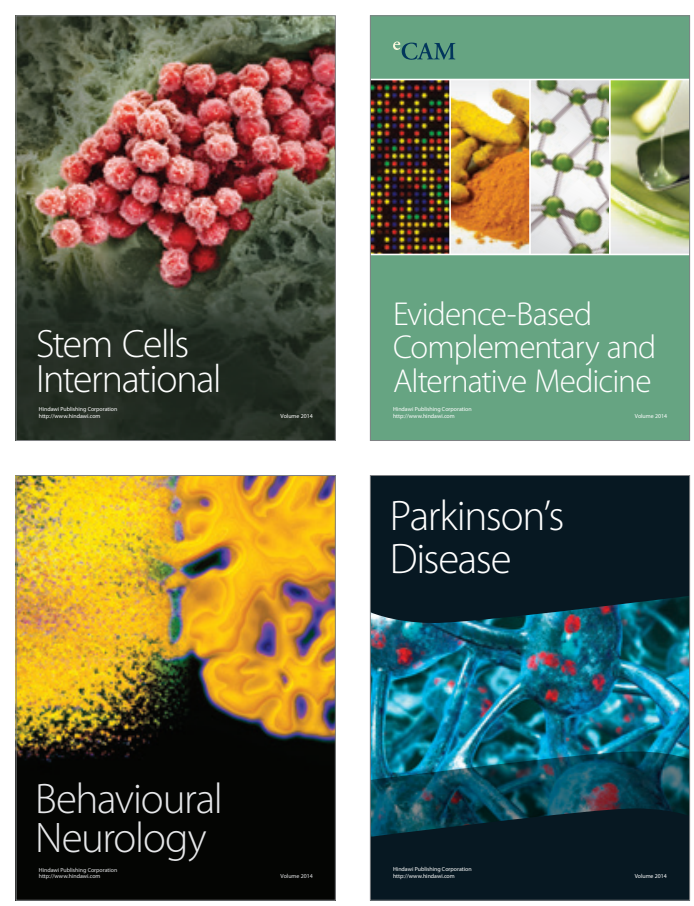

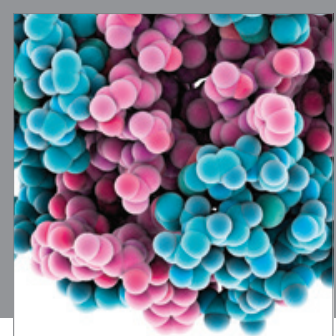

Journal of
Diabetes Research

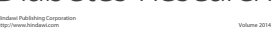

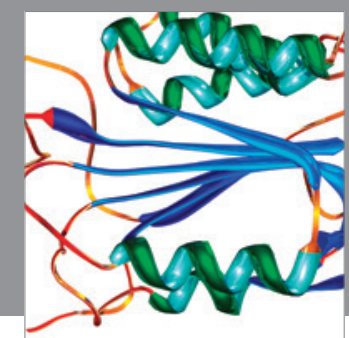

Disease Markers
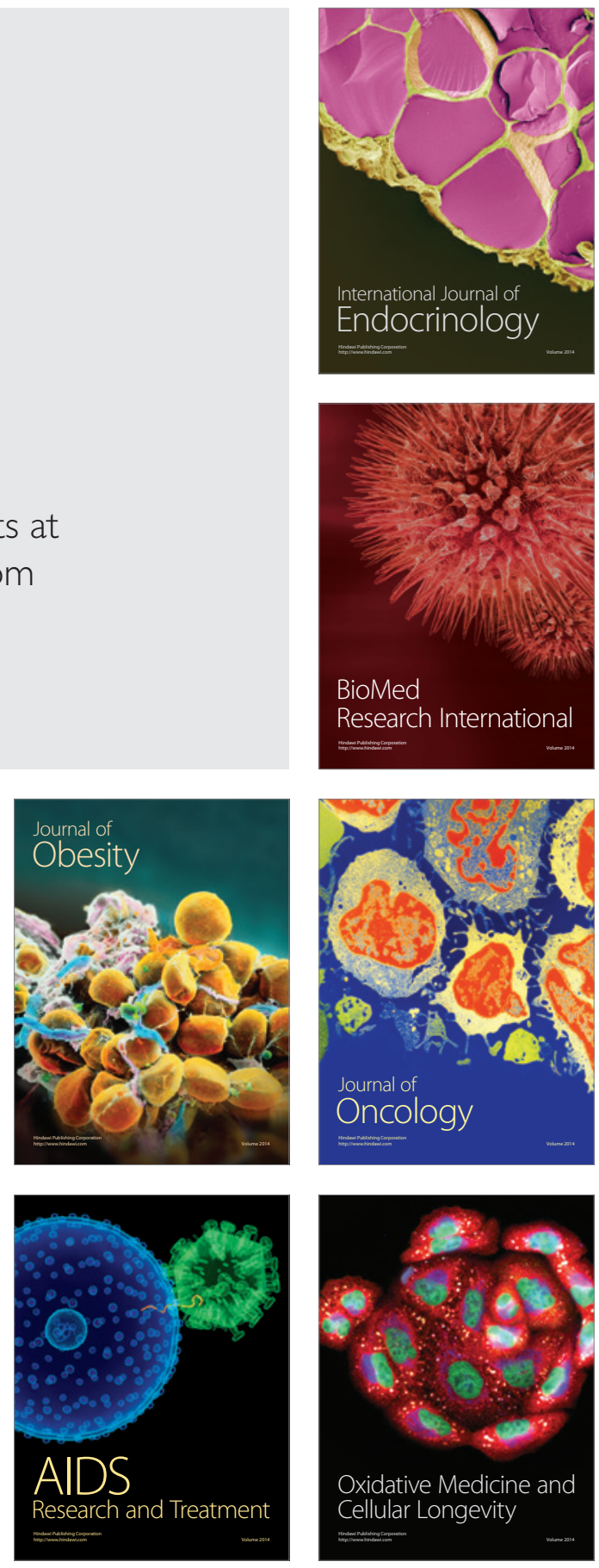